\title{
Enabling Validation of IEEE 802.15.4 Performance through a New Dual-Radio Omnet++ Model
}

\author{
Francisco Jose Estevez ${ }^{1,2}$, Jose Manuel Garcia ${ }^{1,2}$, Jose Maria Castillo-Secilla ${ }^{3}$, Jesus Gonzalez ${ }^{2}$, \\ Peter Gloesekoetter ${ }^{1}$ \\ ${ }^{1}$ Department of Electrical Engineering and Computer Science, University of Applied Sciences of \\ Munster, \\ Germany \\ ${ }^{2}$ Department of Computer Architecture and Technology, University of Granada, \\ Spain \\ ${ }^{3}$ Computer Architecture, Electronics and, Electronic Technology Department, University of Cordoba, \\ Spain \\ fjestevez@ieee.org
}

\begin{abstract}
Internet of Things (IoT) presents a promising technology concept for a wide range of applications due to the possibility of interconnecting any device with connection to Internet in order to improve the daily life of human beings. This fact, along with the increasing use of devices with wireless capabilities has created a saturation problem in the use of the radio-electric spectrum, which decreases the performance of wireless communications. A way to solve this problem is to develop policies for communicating nodes in different spectrum channels by means of the use of a dual radio scheme, which permits the communication in the same network using several channels. In this paper, we evaluate the performance of a dualradio scheme in IEEE 802.15.4 networks for the Ad-Hoc Ondemand Distance Vector routing protocol (AODV). The evaluation of our proposal has been done by developing a new IEEE 802.15.4 dual-radio module for OMNeT++ Network Simulator. Our simulation results show up to $36 \%$ increase of network throughput when dual-radio schemes are used in the nodes, reaching a maximum of $20 \%$ increase in the energy consumption.
\end{abstract}

Index Terms-Wireless sensor network; dual radio; 802.15.4; Omnet++; collisions; AODV.

\section{INTRODUCTION}

Low power communications and low cost hardware are the bases for designing and deploying Wireless Sensor Networks (WSN) [1]. This improvement has also made possible the growing of the concept of IoT, which is a term for identifying the interconnection of "things or objects" independently of its use. Nevertheless, certain challenges exist, which have been researched by the community in order to improve network performance. Among these challenges, lifetime and throughput in terms of sent packets have been highly studied by the research community. The amount of collisions is highly correlated with the throughput of the network because they cut off the communication process between them, forcing to retry the communication process. When density of nodes is increased and they share

Manuscript received 16 September, 2015; accepted 18 February, 2016. the spectrum in the wireless communication process, the number of collisions tends to increase in the same way. How to improve the use of the spectrum when devices with wireless capabilities communicate between them has been a deep field of study by the research community [2], [3].

When the number of wireless devices sending information increases, the number of collisions in the medium increases too reducing the throughput in wireless networks. Therefore, the need for improving the use of radio electric spectrum in order to optimize wireless communications between low cost devices arises. As long as this problem remains unsolved, we consider that we need a model to find effective solutions. In order to achieve this goal, a simulator appears as an obvious solution, in terms of cost and time. Based on simulations, this work is focused on the development of a dual-radio module, which is used in typical high-density scenarios. To develop a reliable module, these test scenarios are based on $A O D V[4]$, giving verifiable results. This work analyses both results, for a single-radio and a dual-radio model, carrying out a detailed comparison.

The main contributions of our work are:

- A new dual-radio module for $O M N e T++$ has been developed, which can be used by the research community in order to develop new tests in the future.

- Based on $A O D V$, it has been analysed how the use of a dual-radio scheme for IEEE 802.15.4 can improve network throughput in terms of collisions and sent packets for high-density scenarios.

- Also, it has been analysed how a dual-radio configuration affects the energy performance.

The rest of the paper is organized as follows: Section II provides an overview of the most relevant related work in dual-radio techniques. Section III shows the system designed for improving collisions and, therefore, the throughput of the networks by means of the use of a dualradio scheme. Simulations and Results obtained with this new proposal are shown in Section IV. Finally, Section V explains the most relevant conclusions of this paper. 


\section{RELATED WORK}

The scientific community has researched in the use of dual radio techniques since 2007 in different works. Several authors have dealt with the challenge of improving Wireless Network performance by means of using dual-channel or/and dual-radio techniques. Among these techniques MIMO [5], which is based on the use of multiple radios in wireless data communication system integrated on a same chip with separated RF circuits separated for each antenna can be highlighted. A real example of the above mentioned technique is IEEE $802.11 \mathrm{n}$, which uses several antennas for $\mathrm{Wi}-\mathrm{Fi}$ communication.

Several authors have proposed dual-radio schemes with great results in terms of throughput:

Kusy et al. [6] found that Wireless Networks throughput improved by using dual radio. In that work the authors compared the performance of dual-band and single-band radio communication using a testbed of 30 nodes. They demonstrate how radio diversity can significantly improve delivery rates, network stability and transmission costs with only a slight increase in energy costs compared to a single radio scheme.

Yin et al. [7] found that networks formed in $900 \mathrm{Mhz}$ band have $15 \%$ more connectivity than network formed in $2.4 \mathrm{GHz}$, even on radio channels that minimize overlaps with Wi-Fi networks. The experiments performed by Yin et al. were set up at multiple channels simultaneously reinforcing the idea that a Dual Channel configuration in Wireless Networks can improve its performance.

Campbell et al. [8], [9] studied multichannel communication based on the 802.11 DCF over a single radio for Wireless Sensor Networks in order to improve its communication performance on throughput, end-to-end delay and channel access delay. They proposed back off algorithm (MC-DCF), which allows nodes to have access to multiple non-overlapping channels by accessing channels dynamically through channel switching after a set threshold has been met.

Pirzada et al. [10] evaluated the performance of the multiradio Ad-hoc On-demand Distance Vector (AODV) routing protocol with a specific focus on hybrid WSNs. In that work they did a set of simulations, showing that under high mobility and traffic load conditions, multi-radio $A O D V$ offers superior performance as compared to its single-radio counterpart.

Jeong et al. [11] consider a scenario in which a set of clusters exists, with a cluster head node in each one equipped with dual radios: IEEE 802.15.4 and IEEE 802.11 for intra-cluster and inter-cluster communication. IEEE 802.11 links provides a high performance backbone for large scale WSN. Both communication protocols work in the $2.4 \mathrm{GHz}$ ISM band. In order to avoid possible communication interferences, they proposed an adaptive aggregation of packets and adaptive transmission scheduling. Results obtained show that their scheme significantly reduces the interferences between the two types of radios.

The novelty of this paper is the development of a dualradio module for $O M N e T++$, which is used to evaluate IEEE 802.15.4 networks' performance. Previous works have studied multi-radio IEEE 802.15.4 and multi-radio AODV, but as we know, never before together. Our proposal is focused on demonstrating how dual-radio can improve IEEE 802.15.4 Wireless Sensor Communications throughput with an $A O D V$ routing scheme, using a new module for OMNeT++.

\section{METHOdOLOGY}

The main idea behind this work was to develop a method to reduce the high number of collisions in WSN with highload traffic. Therefore, thinking about the inclusion of an additional radio that works in a different frequency or channel, could decrease the number of collisions and increase the throughput.

There exist different proposals of dual-/multi-radio schemes as it was commented in Section II, but all of them represent a mid- or high-cost solution, in terms of hardware development costs. Thinking about reaching a major audience, the use of a simulator appears as the most appropriated option.

This work is based on the standard IEEE 802.15.4, because it is the common base for the majority of WSN protocols.

The simulation software used was $O M N e T++$, due to its capacity to simulate a wide range of standard protocols. For WSN, some additional frameworks are available, including INETMANET, a branch of the INET framework that contains the standard model library of $O M N e T++$ and adds several features and protocols, mainly for mobile ad-hoc networks and Wireless Personal Area Networks (WPANs).

$O M N e T++$ implements all the different elements that form a wireless node through modules. These modules are single objects that contain not only the functionality of a full-functional network stack, covering from physical-layer to application-layer, but also the battery, wireless channel control, terminal interface, etc. For this reason, the main objective was to create a module integrating two network interfaces, considering them as the junction of physical radio (PHY) and medium access control (MAC). For a better comprehension, the single-/multi-interface scheme is referred as single-/multi-radio scheme from now on.

As starting point, it was decided to compare both models, single-radio and multi-radio, using a representative example in $O M N e T++$. The need to use a well-known routing scheme leads us to choose AODV. Firstly, because there is an implementation ready-to-use for $O M N e T++$. Secondly, because it is the routing algorithm used by ZigBee and 6LoWPAN (under the LOAD routing), some of the most representative IEEE 802.15.4 standard-based network protocols [12], [13].

As it was commented, the $A O D V$ example exists for $O M N e T++$ as an example called csma802154 (it is included in the wpan examples folder from INETMANET). This network example has several modules like the referent to the User Datagram Protocol (UDP), the Transmission Control Protocol (TCP) or the Internet Protocol (IP) and a routing module for mobile ad-hoc networks (MANETs). Thus, this module allowed us to use $A O D V$ routing.

An important feature of this new module is to keep a high reusability, so the own modules from INETMANET have been modified as few as possible. Keeping this in mind, the IEEE 802.15.4 interface (MAC + PHY) was duplicated as 
shows Figure 1, adding an extra module called cognitiveControl, to handle the compatibility between the regular modules and the dual-radio module.

In order to reach the integration with the existing modules, the original source code was minimally modified, due to the need to register the radio interfaces on the simulator interface list.

The main issue that should be solved was the selection of the radio that should send the data at a certain time. The $M A N E T$ module is responsible of the routing process. It saves the routes between nodes using their IP addresses. That behaviour would not be useful with a dual-radio, since the module owns the same address for both radios and the MANET module could not identify which radio receives/sends the data.

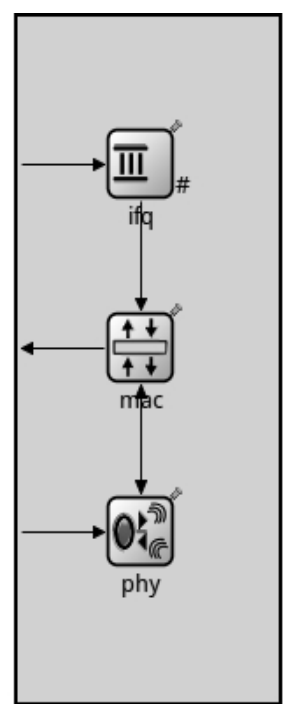

(a)

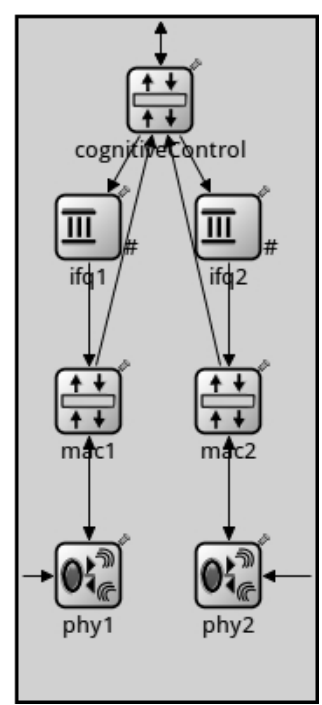

(b)
Fig. 1. $O M N e T++$ modules overview for single-radio scheme (a) and multiradio scheme (b).

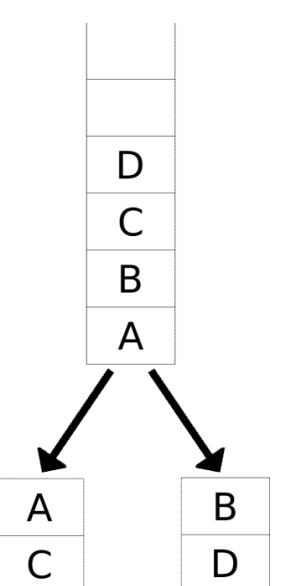

Fig. 2. cognitive Control interface module for OMNeT++.

For that reason, the cognitiveControl module acts as a complement of the MANET module, specifically designed to improve the sending capacity of a node, allowing it sending several packets parallel. In nodes equipped with multiradio, the radio selection is carried out by an algorithm that chooses the radio with lower workload, sending the message to that interface. Figure 2 shows an example of the radio packet distribution. Our module also stores a complementary route according to the radio that should be active in each case. This configuration has been possible through the adaptation of the original IEEE 802.15.4-MAC module. It conserves the entire functionality of the initial module, but if the cognitive module is active, it does not send the data received as airframes directly to the network layer. Instead, data traffic is redirected to the cognitive Control module, and there, the MAC addresses are extracted in order to obtain the necessary information for the routing process. Once the necessary IP and MAC information are known, routes can be established. If the cognitive module is not active, the IEEE 802.15.4 module remains unchanged and works normally.

\section{Simulations AND Results}

The simulations are carried out in an outdoor obstacleand interference-free scenario, where the position is calculated using a random spatial distribution in twodimensional square areas. The propagation model was configured ideally due to the objective in terms of analysis searched by this work. We use a log-normal shadowing propagation model [14] achieving realistic link behaviour using the configuration shown in Table 1. Some of these parameters are the recommended by $O M N e T++$ IEEE 802.15.4 model for MAC and PHY layers.

TABLE I. CONFIGURATION PARAMETERS.

\begin{tabular}{|c|c|}
\hline Parameter & Value \\
\hline Effective Range $(\mathrm{m})$ & 65 \\
\hline Carrier Frequency $(\mathrm{GHz})$ & 2.4 \\
\hline Carrier sense sensitivity $(\mathrm{dBm})$ & -85 \\
\hline Transmit power $(\mathrm{mW})$ & 0.2 \\
\hline Thermal noise (dBm) & -110 \\
\hline Path loss alpha & 2 \\
\hline SNR threshold (dB) & 4 \\
\hline MAC queue length & 50 \\
\hline Routing Protocol & AODVUU \\
\hline Message Length (Bytes) & 70 \\
\hline Multi-radio selected channels & $0 / 5$ \\
\hline
\end{tabular}

After a tuning phase, these simulations were limited to duration of 3600 seconds, due to memory restrictions.

The scenario above details a fixed framework for all the simulations carried out. Table II shows the list of variable parameters with their respective values, which define the different test cases.

TABLE II. SIMULATION PARAMETERS.

\begin{tabular}{|c|c|}
\hline Parameter & Value \\
\hline Number of sending nodes & $5 / 9$ \\
\hline Number of multiple destination nodes & $3 / 4$ \\
\hline Node degree & $5 / 10 / 15$ \\
\hline Time between messages (secs) & $1 / 30$ \\
\hline
\end{tabular}

The time values in which this analysis is based on, were deeply analysed using the mean test and the Kruskal-Wallis test, resulting in a non-correlated scenario, nor for the single-radio model, nor for the multi-radio model. As long as the results obtained are statistically different, the test cases cover the comparison between the two different models: one based in a single-radio and another one based 
on dual-radio. For these radio models, node density is changed (or node degree, ND) simulating 3 different cases: a large network scenario, where ND 5; A medium-size network scenario, with ND 10 and a small scenario with ND 15. All of them are compared under different stress levels. A low-traffic scenario, which involves up to 15 active nodes sending messages every 1 or 30 seconds (it varies depending on the test case); And a high-traffic scenario, which involves up to 40 active nodes, sending every 1 or 30 seconds (it varies depending on the test case).

In order to make a fair comparison between models, three parameters are compared: Error Rate (ER), Packet Delivery Ratio (PDR), and energy consumption. For a better comparison a node density of 3 and a time interval of 0.5 seconds were included in the ER and PDR comparisons.

Figure 3 shows a comparison among different node degrees, different messaging frequency and different models in a low-traffic scenario. A higher value represents a worst performance, due to a higher number of collisions per sending. All the 30 -secs cases show a worst performance of multi-radio against the single-radio model. This time interval allows the single-radio model to handle the workload with a sufficient capacity, whereas the multi-radio model interferes itself, resulting in a higher error rate. On the other hand, for a higher messaging frequency, the multiradio model handles the workload, as it was expected, better than the single-radio model, independently of the node density.

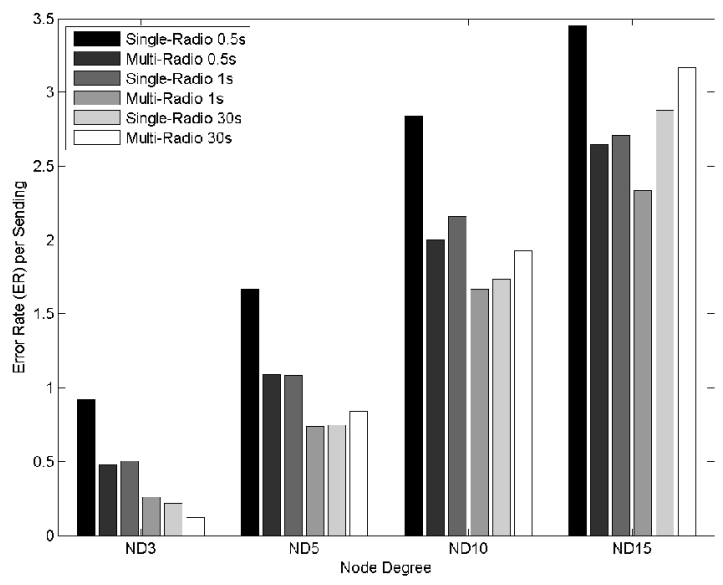

Fig. 3. ER for single-/multi-radio models based on different node degree schemes and different sending frequencies in a low-traffic scenario.

Figure 4 shows the ER for a high-traffic scenario, where only 2 different ND were tested, due to the active nodes requirement for these scenarios. Again, a higher value represents a worst performance. Now the multi-radio model seems to be similar or better for all the configurations in terms of ER.

Figure 5 shows the PDR for both models, considering also the node degree and messaging frequency. This scenario refers to a low-traffic simulation. This time, a higher value represents a better performance. Figure 5 shows interesting results. Under low-traffic conditions, there are three different trends. Firstly, for a higher node density the overall performance drops, independently of the time interval or the configuration. Secondly, for a lower density the single-radio model seems to have a better performance. Thirdly, for a shorter time interval the multi-radio model seems to have a better performance. These two trends meet for the $\mathrm{ND}=3$ and 0.5 seconds case, where both are really similar.

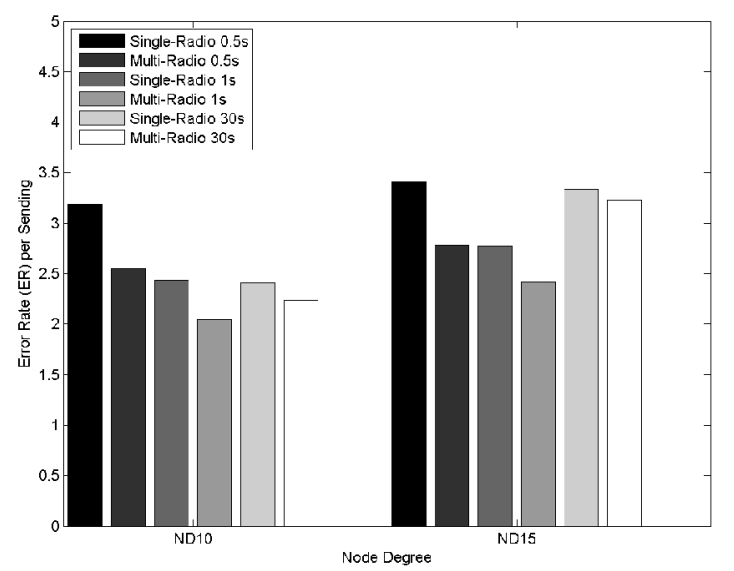

Fig. 4. ER for single-/multi-radio models based on different node degree schemes and different sending frequencies in a high traffic scenario.

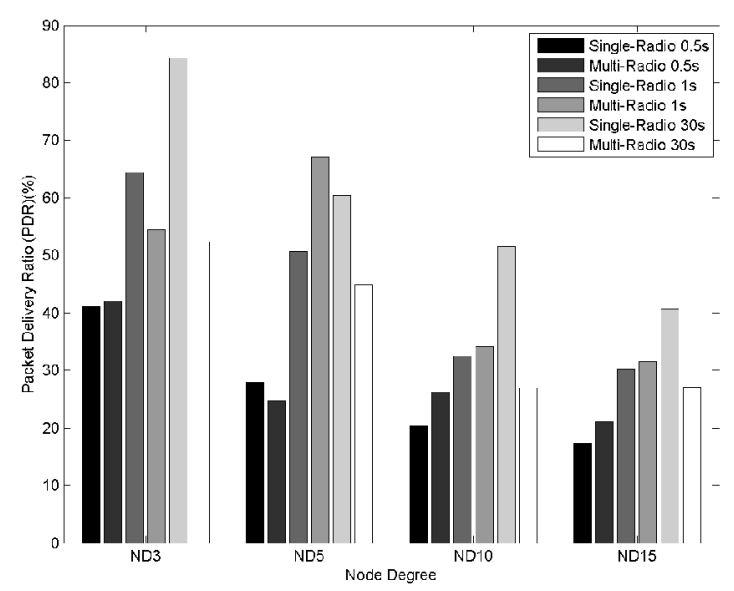

Fig. 5. PDR for single-/multi-radio models based on different node degree schemes and different sending frequencies in a low traffic scenario.

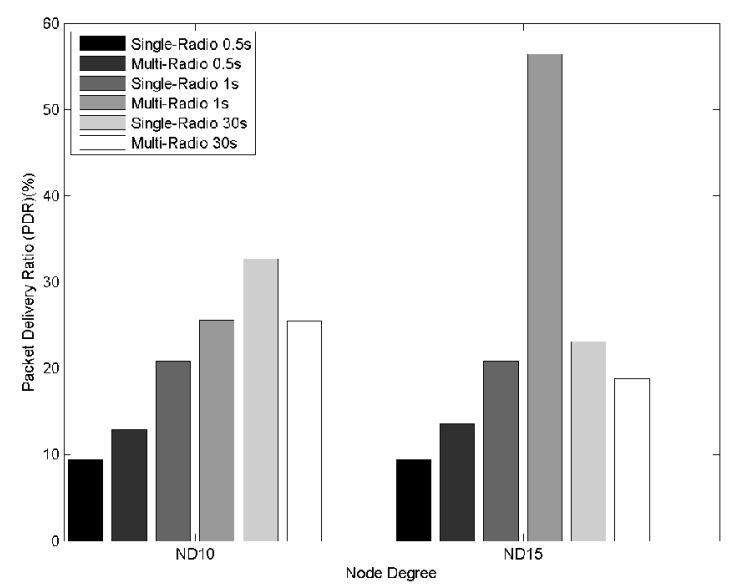

Fig. 6. PDR for single-/multi-radio models based on different node degree schemes and different sending frequencies in a high traffic scenario.

Again, Figure 6 shows a PDR analysis based on ND and messaging frequency for a high-traffic scenario. This chart shows the most interesting result, the performance for a high node degree $(\mathrm{ND}=15)$ and high frequency messaging ( 1 second). The improvement of a dual-radio scheme is far bigger than another one, representing an increment of $36 \%$. The following figures show the energy consumption under different stress levels, due to the different proportions from the node degree scheme, a direct comparison between 
different densities does not provide fair values. Therefore, the energy consumption results are presented as node degree individual, analysing every node degree separately.

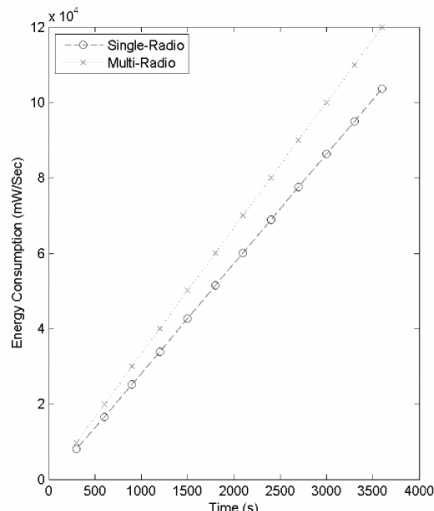

(a)

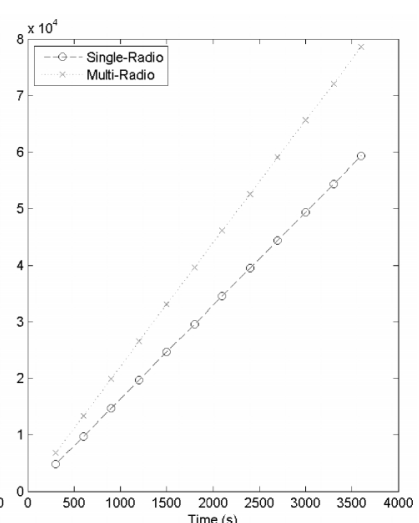

(b)
Fig. 7. Energy consumption for single-/multi-radio models based on ND = 5 at $1-\sec (a)$ and $30-\operatorname{secs}($ b) frequency in a low-traffic scenario.

Figures 7-9 show the energy consumption in $\mathrm{mW} / \mathrm{sec}$ for the low-traffic scenario. Different node degrees at 1 - and 30seconds were respectively analysed.

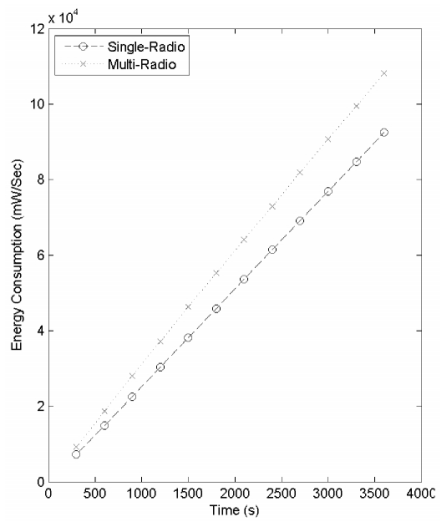

(a)

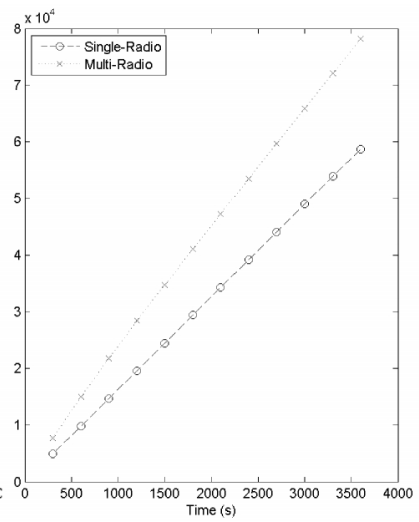

(b)
Fig. 8. Energy consumption for single-/multi-radio models based on $\mathrm{ND}=10$ at 1 -sec (a) and 30 -secs (b) frequency in a low-traffic scenario.

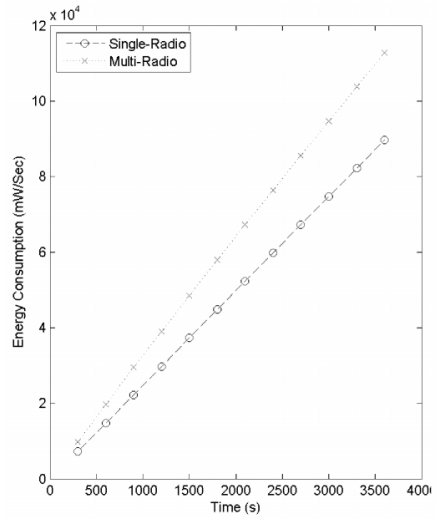

(a)

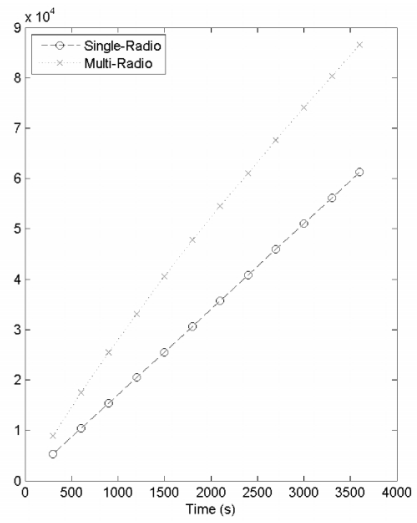

(b)
Fig. 9. Energy consumption for single-/multi-radio models based on ND= 15 at 1 -sec (a) and 30 -secs (b) frequency in a low-traffic scenario.

As it was expected the single-radio model consumes less energy than the multi-radio model. The difference is higher between single- and multi-radio models for the 30 -secs scenario.

This behaviour results consistent with the ER results, where multi-radio at 30 -secs had for all the node degrees a worst performance, resulting in more time switched on and consuming energy. It is also interesting to cross the results of the low-traffic figures. We can see a general higher consumption for 1-sec scenarios, where we can assume that the collisions are higher, making the radios remain active longer times.

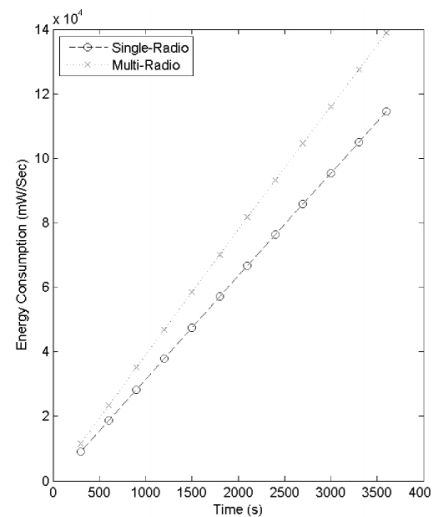

(a)

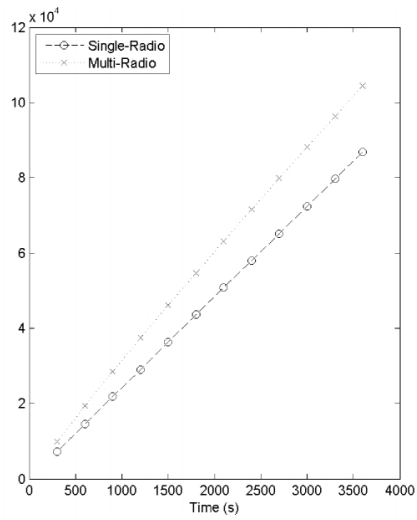

(b)
Fig. 10. Energy consumption for single-/multi-radio models based on ND= 10 at 1 -sec (a) and 30-secs (b) frequency in a high-traffic scenario.

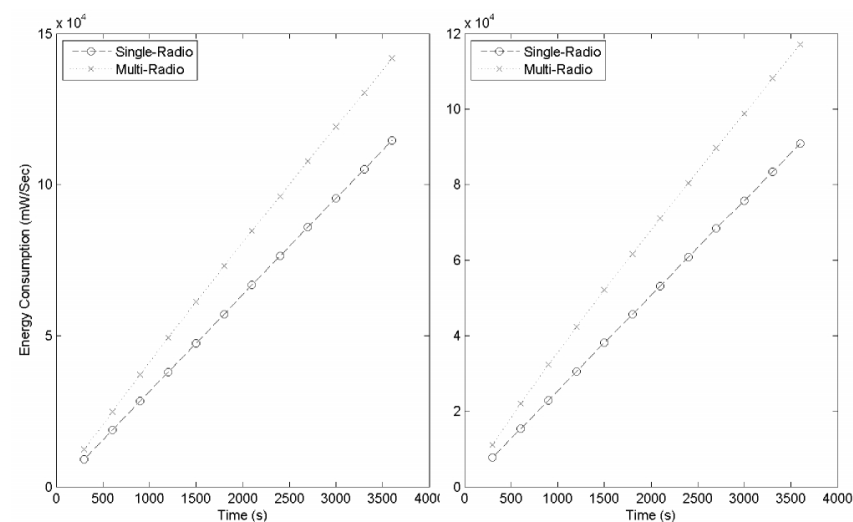

(a)

(b)

Fig. 11. Energy consumption for single-/multi-radio models based on ND= 15 at $1-\sec (a)$ and $30-\operatorname{secs}($ b) frequency in a high-traffic scenario.

Figure 10 and Fig. 11 present the results in terms of energy consumption in $\mathrm{mW} / \mathrm{sec}$ for the high-traffic scenario. Both models were tested in different node densities and time intervals, presenting interesting results.

As it was expected the used energy is higher for $\mathrm{ND}=15$ than ND $=10$. However it is possible to see in details the energy difference between $\mathrm{ND}=10$ and $\mathrm{ND}=15$, reaching this increment a value between a $15 \%$ and $25 \%$.

Based on the previous analysis, it can be observed a trend where the gain from the density increment in high-traffic scenarios tends to be higher than the energy consumption increment, due to the reduction on the number of collisions and the reduction on the active time of the radios. This fact reveals the benefits of using two radios. Of course, this involves a higher hardware cost, but depending on the scenario and the radio features, the gain could be much higher.

\section{CONCLUSIONS}

In this paper we validated by simulation, the influence of two radios in WSN. We also proposed a new dual-radio module for $\mathrm{OMNeT++}$, expanding its capacities for WSN 
simulation.

Results show that using two radios in high-traffic scenarios with a high messaging frequency, improve the error rate and packet delivery ratio in terms of performance, regardless the node density variations. However, the improvement in terms of error rate is lower for scenarios where the node density remains under a 10-degree. Under low-traffic conditions or low messaging frequency, there are no remarkable improvements or even a downturn in terms of performance. The energy consumption is also measured for these different analyses, and it clearly shows a disadvantage for the dual-radio configuration, due to the energy requirement of both radios. The energy increment varies depending on the configuration, but for the best test case, where the performance increment reached up to $36 \%$ in terms of PDR, the energy increment remained in the range of $15 \%-25 \%$. For a hierarchical network configuration based on clustering should be possible to reduce even more the number of collisions, resulting in a better use of the channel and a bigger energy reduction.

Finally, a future work should go deeply into the radio duty cycle management, using a better politics or a smart routing algorithm or protocol. The use of a specific wireless routing algorithm allows managing both radio interfaces, adjusting the wake-up/sleep cycles as much as possible, reducing the energy consumption. With a smarter politic of use, it is possible to remain closer to the single-radio model, improving the throughput and error rate, at the same time that the energy remains nearly constant, representing an improvement for IoT scenarios. Future experiments will be focused on different environment conditions such as indoor scenarios, dynamical obstacles and different radio interferences, testing another propagation models as well.

\section{ACKNOWLEDGMENT}

This work has been partially funded by project TIN201567020-P, granted by the Spanish "Ministerio de Economía y Competitividad".

\section{REFERENCES}

[1] J. Stankunas, D. Rudinskas, E. Lasauskas, "Experimental research of wireless sensor network application in aviation", Electronika ir Elechtrotechnika, vol. 111, no. 5, 2011.

[2] G. Joshi, S. Nam, S. Kim, "Cognitive radio wireless sensor networks: applications, challenges and research trends", Sensors, vol. 13, no. 9, pp. 11196-11228, 2013. [Online]. Available: http://dx.doi.org/ $10.3390 / \mathrm{s} 130911196$

[3] J. Zhu, C. Lv, Z. Tao, "Performance analyses and improvements for IEEE 802.15.4 CSMA/CA scheme in wireless multihop sensor networks based on HTC algorithm", International Journal of Distributed Sensor Networks, 2013. [Online]. Available: http://dx.doi.org/10.1155/2013/452423

[4] IETF RFC3561, AODV Specification: [Online]. Available: https://www.ietf.org/rfc/rfc3561.txt

[5] A. J. Paulraj, D. A. Gore, R. U. Nabar, H. Bolcskei, "An overview of MIMO communications - a key to gigabit wireless", in Proc. the IEEE, 2004, vol, 92, no. 2, pp. 198-218. [Online]. Available: http://dx.doi.org/10.1109/JPROC.2003.821915

[6] B. Kusy, C. Richter, H. Wen, M. Afanasyev, R. Jurdak, M. Brunig, D. Abbot, C. Huynh, D. Ostry, "Radio diversity for reliable communication in WSNs", in 10th Int. Conf. Information Processing in Sensor Networks (IPSN), 2011. Chicago, Illinois, pp. 270-281.

[7] S. Yin, O. Gnawali, P. Sommer, B. Kusy, "Multi channel performance of dual band low power wireless networks", in Proc. 11th Int. Conf. Mobile Ad-Hoc and Sensors Systems (IEEE MASS 2014), 2014. [Online]. Available: http://dx.doi.org/10.1109/mass.2014.120

[8] C. Campbell, K. Loo, R. Comley, "A new MAC solution for multichannel single radio in wireless sensor networks", (ISWCS 2010), 2010, pp. 907-911. [Online]. Available: http://dx.doi.org/10.1109/ iswcs. 2010.5624520

[9] C. Campbell, K. Loo, O. Gemikonakli, S. Khan, D. Singh, "Multichannel distributed coordinated function over single radio in wireless sensor networks", Sensors, vol. 11, pp. 964-991, 2011. [Online]. Available: http://dx.doi.org/10.3390/s110100964

[10] A. A. Pirzada, M. Portmann, J. Indulska, "Performance analysis of multi-radio AODV in hybrid wireless mesh networks", Computer Communications, pp. 885-895, 2008. [Online]. Available: http://dx.doi.org/10.1016/j.comcom.2007.12.012

[11] Y. Jeong, J. Kim, S. Han, "Interference mitigation in wireless sensor networks using dual heterogeneous radios", Wireless Networks, vol. 17, no. 7, pp. 1699-1713, 2011. [Online]. Available: http://dx.doi.org/10.1007/s11276-011-0373-4

[12] ZigBee Alliance, ZigBee Specification Document 053474r17.

[13] 6LoWPAN-LOAD routing algorithm, [Online]. Available: https://tools.ietf.org/html/draft-daniel-6lowpan-load-adhoc-routing03 .

[14] Wireless Communnications and Networks: 3G and Beyond. ITI Saha Misra. McGraw-Hill. 2009. 